\title{
The Analysis of the Dialect Word "De" in Tongchuan District of Dazhou
}

\author{
Ran Ya, Xiao Jiugen* \\ Jiangxi Normal University, Research Center of Language and Language Life, Nanchang, China
}

\section{Email address:}

2713517977@qq.com (Ran Ya),jxsdxjg666666@sina.com (Xiao Jiugen)

* Corresponding author

\section{To cite this article:}

Ran Ya, Xiao Jiugen. The Analysis of the Dialect Word "De" in Tongchuan District of Dazhou. Science Innovation. Vol. 6, No. 4, 2018, pp. 177-181. doi: 10.11648/j.si.20180604.11

Received: April 20, 2018; Accepted: June 22, 2018; Published: July 20, 2018

\begin{abstract}
The Tongchuan district of Dazhou is located in the east of Sichuan province where the dialect belongs to Chengdu-Chongqing area of the southwestern Mandarin.Through the investigation and analysis, we found that the pronunciation, the meaning and grammatical features of Tongchuan dialect word "De" are quite different from those of Chengdu-Chongqing area of the southwestern Mandarin and common language. In Tongchuan dialect, the pronunciation, the meaning and the parts of speech of "De" with high usage rate are different. Moreover, the sentence pattern choice is very flexible, and the sentence pattern is diversified.We analyze the meaning of pronunciation and grammatical phenomenon of "De", which aims to show its distinctive regional characteristics, and preliminarily reveal the lexical meaning and grammatical functions of its different parts of speech and various sentence pattern.
\end{abstract}

Keywords: Dialect Words "De", Pronunciation and Meaning, Grammatical Functions, Regional Characteristics

\section{达州通川区方言词“得”的分析}

\section{再娅, 肖九根 ${ }^{*}$}

江西师范大学, 语言与语言生活中心, 南昌, 中国

\section{邮箱}

2713517977@qq.com (申娅),jxsdxjg666666@sina.com（肖九根）

摘要：达州市通川区位于四川省东部，其方言属于西南官话成渝片。通过调查分析,我们发现通川方言词“得”的音义语 法特征与官话成渝片及共同语均存有较大的不同。在通川方言中，使用率很高的“得”读音不同，语义不同，词类分属 也不同; 而且, 其句型选择十分灵活, 句式呈现出多样化态势。我们分析描写“得”的音义语法现象, 旨在展现其鲜明 的地域特色，初步揭示其不同词类及多样化句式所具有的词义和语法功能。

关键词：方言词“得”, 音义, 语法功能, 地域特色

\section{1. 引言}

达州市位于四川东北部, 辖2个市辖区（通川区、达 川区）、4个县（宣汉县、开江县、大竹县、渠县）、1 个县级市 (万源市) 、一个经济开发区 (46个行政村) [1]。
达州方言属于西南官话成渝片一一成 (都) 渝 (重庆) 话, 以成都话和重庆话为代表[2]。由于历史地理等原因, 达州话形成了一些独有的特点, 达州话又叫“平话”、“四平 话”或“四平腔”。达州人口复杂，690万人口。本文研究的 方言主要是达州通川区流行的口头语, 其例句源于我们对 
通川区的语言调查, 虽然也参考了诸如张大旗 (1985) [3] 徐阳春（1998）[4]、陆镜光（1999）[5]等学者关于湘赣粤 方言“得”的用法, 还有其他学者如邓英树 (2013) [6]等对 川省方言“得”的考察，但其研究大都与通川方言词“得”的 用法相去甚远或颇存差异。因此, 我们认为很有必要对其 作进一步考察。对于方言词“得”的研究, 我们主要从其读 音入手，这样似乎更便于分析它的词义和语法特征。

\section{2. “得”的用法}

\section{1. “得 $\left[\operatorname{tie}^{214}\right]$ ”}

达州通川区“得”, 读上声时既可作助动词, 也可作结 构助词。

\subsection{1. 作助动词}

“得 $\left[\mathrm{tie}^{214}\right]$ ”作助动词时, 不同情况表示不同的意义。 具体来说有四:

一是表示条件成熟, 应做某事。如通川家长常对孩子 说: “你走得了, 不然要迟到了。”这个“得”作助动词, 意 即“你该走了, 不然就要迟到了”。

二是表示有能力、善于做某事。例如: “他太喝得酒 了”, 意即“他太能喝了, 喝酒很厉害”。

三是表示许可或请求允许之意, 但值得注意的是一般 用于否定句，意为“不允许做某事”，极少用于肯定句。例 如: “你不得睡觉”是说“你不可以睡觉”, 或“你不准睡觉”, 但没有“你得睡觉”这种表肯定的说法。

四是表示预计某事发生的可能性[7]。例如: “他不得 来了”，表示“他不会来了”。

在通川区人们的日常生活中, 表示可能、意愿、许可 等义, 虽然使用“能、能够、可以、会”等助词, 但更多的 是使用“得”及含“得”的词语 $[8]$ 。

\subsection{2. 作结构助词}

“得”作结构助词时无实际意义，与共同语“得”的用法 一样, 表示附加成分和中心语之间的结构关系, 一般放在 补语前。

\section{2. “得 $\left[\operatorname{tie}^{35}\right]$ ”}

通川区“得”读阳平时, 可作动词。

其一，表示“获取”、“满意”、“实现”义，与共同语动 词“得 $\left[\mathrm{te}^{35}\right]$ ”的用法相同, 仅是读音上的差异。

其二, “得”能单独作谓语, 表示“有”义, 具体将在“得 $+O$ ”句式中分析，不同于共同语的用法。

\section{3. “得[tie]” (轻声)}

通川“得”读轻声时, 既可作语气助词, 也可作词缀。

\subsection{1. 作语气助词}

根据语气程度的不同, 可以分为以下三种: 例如:

其一, 在句末加一个语气助词“得”, 表示陈述语气[9]。

甲：你把书放在哪了？
乙: 搁在书包头得。（放在书包里了。）

其二, 与“哦”连用, 放在句尾, 表示一般强调的语气。 例如: “他好切得哦”, 强调“他太能吃了”, 这里的“切”是 通川方言, 表示“吃”的意思。

其三, 与“嘛”连用, 放在句尾, 一般说话者情绪激动 时使用, 语气强烈。例如:

甲: 这画好看得, 嘛, 你莫把它毁了。

乙: 恩, 晓得 2 了。

“得 1 ”属于此种情况, “得 2 ”则是词缀。甲在说这句话 时, 语气很强, 表示“这幅画非常好看, 不要把它毁了”。

\subsection{2. 作词缀}

“得”作词缀时，可以分为几种情况：

其一, 与共同语的构词成分“得”相同, 可以构成动词, 如“晓得、省得、免得”等。这里的“得”作构词成分后缀, 但前面的词根是不能独立运用的动词性词根, 是一个不成 词语素, 必须与“得”构成动词后才能运用, 而整个词义基 本上还是词根的意义, 作为词缀的“得”不改变词性和意义, 仅起构词作用[10]。

其二, “得”作固定词语中的词缀, 其中最常见的有如 下几种情况:

（1）“喜幸得”表示“幸好、幸亏”之意。例如: “喜幸 得我出去了, 不然挨打的就是我。”

(2)“难得”也同“懒得”, 它与共同语的“难得”不一样, 表示“很不易”、“不愿去做某事”。例如: “他的事情太难得 搞了”, 即是“他的事情做起来太不容易了”; “我难得去他 家了”, 也就是“我不愿意去他家”。

\section{3. “得”的句式选择及句法特征}

通川方言“得”的句式主要有这几类: $V / a d j+$ 得、 $V+$ 得 $+\mathrm{O} 、 \mathrm{~V} / \mathrm{adj}+$ 得 $+\mathrm{C}$ 、得 $+\mathrm{V}$ 等。

\section{1. “ $/ \mathbf{a d j}+$ 得”}

关于“V/adj+得”句式，可从肯定式、否定式及副词选 择等方面分析。

\subsection{1. “ $V+$ 得”肯定式}

“ $\mathrm{V}+$ 得”句式, 根据 $\mathrm{V}$ 的不同类型, 分以下五种情况: 其一, $\mathrm{V}$ 是行为动词, 即“行为动词 + 得”句式, 其中表 动作行为的有: 跑、骂、走、批评等。如通川方言中常听 到“你好睡得哟”类似的句子，意即“你太能睡了”。

其二, $\mathrm{V}$ 是趋向动词，即“趋向动词 + 得”句式，其中表 趋向动词的有: 来、进、出、来、进去等, “趋向动词 + 得”一般表示能干某事。例如:

甲: 下面安不安全, 我能下去吗?

乙: 下来得。

这儿的“下来得”就表示“下面安全, 你能下来”。

其三, $\mathrm{V}$ 是现存动词, 但现存动词仅限于“有”, 而且 仅是“有十不得”这个固定句式, 有不惜某物的含义。例如:

甲: 妈妈, 我把这个衣服扔到垃圾桶了。

乙: 你看啥子东西有不得。 
对话中, 妈妈说: “你看啥子东西有不得”, 意即“太 铺张浪费了, 不懂珍惜”, “看啥子东西”是当地方言, 意 为“不管什么东西”。

其四, $\mathrm{V}$ 是能愿动词, 即“能愿动词 + 得”句式, 能愿动 词仅限于“要”, 构成“要得”、“要不得”、“要不要得”三种 固定搭配，意为“可以”、“不可以”、“可不可以”。例如:

甲: 今天我们出去逛街嘛, 要不要得?

乙: 要得。

其五, 在“ $\mathrm{V}+$ 得”的句式中, $\mathrm{V}$ 不可以是判断动词、心 理动词。判断动词如“是”, 心理动词“喜欢”、“想念”等。

\subsection{2. “adj+得”肯定式}

“adj+得”句式中, “adj”一般有活用现象, adj活用为 V。 如“他好苦得”, 意即“他好能吃苦”。而那些不能活用为 $\mathrm{V}$ 的adj, 则不用“adj+得”句式。

\subsection{3. “V/adj+得”否定式}

其一, “ $\mathrm{V}+$ 得”否定式, 即“ $\mathrm{V}+$ 不得”式。在否定式“ $\mathrm{V}+$ 不得”中, 当主语是受事时, 表示“不许做某事”; 如是施 事时, 则表示“不许做某事”, 或“没有能力做某事”。例如:

A. 这个苹果吃不得。

$B$. 他现在睡不得。

A句仅表示“这个苹果已经不能吃了”; 而B句则有两 个含义: “他睡眠不好, 现在睡不着”以及“现在还不允许 他睡觉”。对于 $B$ 句的歧义现象, 要在具体语境中分析, 才 能确定其准确的含义。

其二, “adj+得”否定式与“ $V+$ 得”的否定式一样，即“adj+ 不得”表示“不能怎样”。如“他苦不得”, 表示“他不能吃苦”。

\subsection{4. “V/adj+得”句式的副词选择}

当主语是施事时, $V / a d j$ 可以受副词修饰, 是受事时则 不能。主语是施事时, “V/adj+得”句式的副词选择可分为 以下两方面:

其一, “V/adj+得”肯定式, 只受程度副词“很”、“好”、 “太”、“硬是”的修饰，其中“硬是”在这表示一种程度，而 且是其中表示程度最深的。如“他硬是喝得”, 即表示“他 实在是太能喝了”。

其二, “V/adj+得”否定式, 只受程度副词“太”、“硬是”、 “一点儿都”修饰, 不能受程度副词“很”“好”修饰。如当地 人常说“我最近一点儿都跑不得”, 却没有“我最近很跑不 得”这种表达方式。

\section{2. “ $V+$ 得 $+O ”$}

“ $\mathrm{V}+$ 得 $+\mathrm{O}$ ”句式可从肯定式、否定式、副词选择以及 宾语前置方面分析。通川方言没有“adj+得 $+O$ ”句式。

\subsection{1. “ $V+$ 得 $+O$ ”肯定式}

“ $\mathrm{V}+$ 得 $+\mathrm{O}$ ”肯定式, $\mathrm{V}$ 有以下三方面的条件和特点:

其一, $\mathrm{V}$ 行为动词, 即“ $\mathrm{V}+$ 得 $+\mathrm{O}$ ”句式, 常见的如“做”、 “喝”、“说”等动词。例如: “你太喝得酒了”、“你太花得钱 了”, 表示“太能喝酒”、“太能花钱”。

其二, $\mathrm{V}$ 趋向动词, 即“ $\mathrm{V}+$ 得 $+\mathrm{O}$ ”句式, 常见的如“去”、 “来”等。例如: “已经七点半了, 你该去得学校了。”
其三, “ $\mathrm{V}+$ 得 $+\mathrm{O}$ ”句式中, 不能用现存动词、能愿动 词、判断动词、心理动词, 这些动词日常交流中, 不符合 人们的交际习惯。

\subsection{2. “ $V+$ 得 $+O$ ”否定式}

“ $\mathrm{V}+$ 得 $+\mathrm{O}$ ”否定式, 有以下两种情况:

其一, “得”前加“不”, 即“ $\mathrm{V}+$ 不得 $+O$ ”句式。值得注 意的是, 当 $\mathrm{V}$ 是行为动词时, “ $\mathrm{V}+$ 不得 $+\mathrm{O}$ ”句式会产生歧义, 表示“不能做某事”或“不擅长做某事”。例如: “他吃不得辣 椒”这句话, 表示两种意思:

A、由于身体原因, 他不能吃辣椒。

B、他不太会吃辣椒。

其二, “ $\mathrm{V}+$ 得 $+\mathrm{O}$ ”否定式, 在 $\mathrm{V}$ 前加否定副词“不好”, 构成“不好 $+\mathrm{V}+$ 得 $+\mathrm{O}$ ”否定式。但是, 否定式“不好 $+\mathrm{V}+$ 得 $+\mathrm{O}$ ”中的 $\mathrm{V}$ 仅限于“骂”、“打”、“批评”等带主观色彩的动词。 如家长常对孩子说: “我不好打得你”, 意即“我给你点面 子, 不想打你”。

\subsection{3. “ $V+$ 得 $+O$ ”句式的副词选择}

“ $\mathrm{V}+$ 得 $+\mathrm{O}$ ”句式的副词选择, 可以分以下两方面:

其一, “ $\mathrm{V}+$ 得 $+\mathrm{O}$ ”肯定式, 只受程度副词“很”、“好”、 “太”、“硬是”修饰, 其中“硬是”在这儿表示一种程度, 而 且是表程度最深的。如“你太做得活路了”, 就表示“你太 能干活了”, 其中“活路”是通川方言词, 就是“干活”的“活 儿”。

其二, “ $\mathrm{V}+$ 得 $+\mathrm{O}$ ”否定式, 只受程度副词“很”、“太”、 “硬是”、“一点儿都”修饰, 不受程度副词“好”的修饰。但 是, 程度副词“很”修饰否定式“不好 $+\mathrm{V}+$ 得 $+\mathrm{O}$ ”, “一点儿 都”修饰否定式“ $\mathrm{V}+$ 不得 $+\mathrm{O}$ ”。例如:
$A$ 、我很不好批评得你。
$B 、$ 他一点儿都吃不得亏。

$\mathrm{A}$ 句和 $\mathrm{B}$ 句是人们常用的表达方式, 却没人说“我一点 儿都不好批评得你”, 也没人说“他很吃不得亏”。可以受 程度副词“太”、“硬是”的修饰, 如有“我硬是不好批评得你” 类似的说法。

\subsection{4. “ $V+$ 得 $+O ”$ 句式的宾语前置情况}

“ $\mathrm{S}+\mathrm{V}+$ 得 $+\mathrm{O}$ ”结构中, 宾语可以前置, 放在句首充当 话题, 构成“ $\mathrm{O}+\mathrm{S}+\mathrm{V}$ 得”这一句式[11]。如“饭你太切（吃） 得了”, “饭”作为受事名词性成分, 虽然提于句首, 但整 个句义不变, 表示“你太能吃了”。

如果把“ $\mathrm{S}+\mathrm{V}+$ 得 $+\mathrm{O}$ ”结构记作 $\mathrm{S}_{1}$, “ $\mathrm{O}+\mathrm{S}+\mathrm{V}+$ 得”式则可 记作 $\mathrm{S}_{2}$ 。 $\mathrm{S}_{1}$ 和 $\mathrm{S}_{2}$ 之间的转换是有条件的, 即要满足以下三 个条件:

第一, “S”必须是“V”的直接施事; 第二, “O”必须是“V” 的直接受事; 第三, 在实际运用过程中, “V”和“ $O$ ”不能是 凝固在一起的词或惯用语[7]。

下列句例不符合上述条件, 均不能实现 $\mathrm{S}_{1}$ 和 $\mathrm{S}_{2}$ 之间的 转换:

A、这个轮船太承载得客人了。—客人这个轮船太 承载得了。(现在的轮船太能承载客人了。)

$\mathrm{B}$ 、这个玻璃瓶太塞得东西了。—东西这个玻璃瓶 太塞得了。（这个玻璃瓶太能装东西了。） 
C、你太睡得瞌睡了。—瞌睡你太睡得了。（你太 能睡觉了。)

$D$ 、我爸最吹得牛皮了。一牛皮我爸最吹得了。( 我 爸最能吹牛皮了。)

E、王叔叔太游得泳了。— 泳王叔叔太游得了。（王 叔叔太能游泳了。）

$\mathrm{A}$ 句的“客人”不是“承载”的受事, 是“承载”的施事, “这个轮船”才是被“承载”, 这句话的语义关系应为“客人在 这个轮船上”，不符合第一个条件，所以“客人”不能放到 句首。B句的“玻璃瓶”不是“塞”的直接施事, 不符合第二 个条件，所以“东西”不能放至句首。C句的“睡觉”、D句的 “吹牛皮”和 $E$ 句的“游泳”是惯用词，不能分开使用，也不 符合第三个条件, 所以不能放到句首。

\section{3. “V/adj+得 $+\mathbf{C} "$}

“V/adj+得 $+C ”$ 句式也可从肯定式、否定式、副词选择 三方面分析。

\subsection{1. “ $V+$ 得 $+C$ ”肯定式}

“ $\mathrm{V}+$ 得 $+\mathrm{C}$ ”句式, $\mathrm{V}$ 的不同分为以下四种情况：

其一, $\mathrm{V}$ 行为动词, 即“ $\mathrm{V}+$ 得 $+\mathrm{C}$ ”句式, 表行为的动词 有: “睡”、“骂”、“走”、“看”等, 意味着某件事可能发生 或即将发生, 强调事件的结果。吕叔湘、朱德熙认为, 就 意义方面说凡是动词后面的附加语都有表示动作结果的 意思, 用“得”字连接的尤其明显[12]。比如说, 通川方言 中, 常听到“他拿得起来”类似的句子, 表示“他能拿起来”。

其二, $\mathrm{V}$ 趋向动词, 即“ $\mathrm{V}+$ 得 $+\mathrm{C}$ ”句式, 其中表趋向的 动词有“来”、“进”、“出”、“来”、“进去”等。例如:

甲: 小明已经去学校了吗?

乙: 嗯, 他去得太早了。

其三, $\mathrm{V}$ 心理动词, 即“ $\mathrm{V}+$ 得 $+\mathrm{C}$ ”句式, 表心理的动词 有“怕”、“恨”、“觉得”、“爱”等, 这与共同语的用法相同。 例如:

甲：你班主任上次骂了你，你恨他吗？

乙: 不恨, 相反, 喜欢得很。

其四, 在“ $\mathrm{V}+$ 得 $+\mathrm{C}$ ”句式中, $\mathrm{V}$ 不可以是判断动词“是”, 也不能是情愿动词“愿意、“应该”、“能”和存现动词“有”、 “在”等。

\subsection{2. “ $a d j+$ 得 $+C$ ”肯定式}

“adj+得 $+C$ ”句式中, “adj”一般能活用为 $V$ 。如“她乘得 很”, 意即“她长得好漂亮”, “乘”这个词在通川方言中表示 “漂亮”。

\subsection{3. “V/adj+得 $+C$ ”否定式}

“V/adj + 得 $+C$ ”否定式, 其中“adj + 得 $+C$ ”没有否定式, 只有“ $\mathrm{V}+$ 得 $+\mathrm{C}$ ”有否定式。“ $\mathrm{V}+$ 得 $+\mathrm{C}$ ”否定式是在“得”后加 “不”, 即“ $V+$ 得 + 不 $+C$ ”, 并且句式“ $V+$ 得 + 不 $+C$ ”中的 $V$ 限 于行为动词、趋向动词。例如:

甲: 他的毛笔字写完了吗?

乙: 写完了, 但写得不好。

这儿的“写得不好”就是否定式“ $\mathrm{V}+$ 得 + 不 $+\mathrm{C}$ ”。

\subsection{4. “V/adj + 得 $+C$ "句式的副词选择}

“V/adj+得 $+C ”$ 的副词选择, 其中“adj+得 $+C$ ”本来表示 某种程度, 不能受副词修饰, 只有“ $\mathrm{V}+$ 得 $+C$ ”句式有副词 选择。“ $V+$ 得 $+C$ ”对副词的选择, 可归纳为以下三种形式:

其一, “ $\mathrm{V}+$ 得 + (程度副词) 太、好 $+\mathrm{C} ”$, 这种句式是把 程度副词“太”、“好”放在 $C$ 前。如“你想得好美哦”, 就是把 程度副词“好”放在补语“美”之前, 表示“你想得太好了”。

其二, “硬是 $+\mathrm{V}+$ 得 $+\mathrm{C}$ ”, 程度副词“硬是”只能放在 $\mathrm{V}$ 前。如“他硬是长得丑”, 却没人说“他长得硬是丑”, “硬是” 在这表示一种较深程度, 是“极其”之意。

其三, “ $\mathrm{V}+$ 得 + 不 $+\mathrm{C}$ ”这种否定式只能在“不”前加程度 副词“太”, 即构成“ $\mathrm{V}+$ 得 + 太 + 不 $+C$ ”这种句式。例如:

甲: 他的字写得怎么样?

乙: 他写得太不好看了。

\section{4. “得 $+V$ ”}

\subsection{1. “得 $+V$ ”肯定式}

这里的“得”作为助动词, 表示“可能”的意思, 即是客 观可能性，相当于“可能、会”。“得”字不仅可以放在动词 后面, 还可以放在动词前面, 这里的动词包含了行为动词、 心理动词、趋向动词、能愿动词。值得注意的是, 通常要 在“得”字前加上“才”, 构成“才得”, 类似于“才会、才可能 做什么”的意思。例如:

A、他只要不扯皮, 才得出去要。（他只要不顽皮, 才能够出去玩。)

B、你睡完午觉起来才得吃饭。（你睡完午觉起来才 能吃饭。)

C、吃完药以后才得去看电影。（吃完药才能去看电 影。)

\subsection{2. “得 $+V$ ”否定式分析}

“得 $+V$ ”的否定式是“不得 $+V$ ”[13]。例如:

A、大姑没回来，你就不得走了。（大姑没回来，你 就不能离开。)

$\mathrm{B} 、$ 你不睡午觉不得吃饭。(你不睡午觉就不能吃饭。)

C、天气变凉了, 不得吃冰淇淋。（天气变凉了, 不 能吃冰淇淋。)

从以上例句可以看出, 它们有一个共同格式: “不... 不得”, 这实质上是一个条件句, “不...”是“不得”的一个条 件。如“小朋友不等到十一点不得睡觉”这句话, “十一点” 就是“他睡觉”的一个充要条件, 不符合“十一点”这个条件, 他就“不得睡觉”; 相反, 只有符合了“十一点”这个条件, 小朋友“才得睡觉”。从这可知, “才得”相当于“只有...才” 句式, 那么“不...不得”相当于普通话的“若不...就不”句式。

\subsection{3. 固定搭配“得行不得行”与“得不得 $+V$ ”}

其实, 日常生活中, 人们还常用“得行不得行”及“得 不得 $+V$ ”两种固定搭配来征求对方意见, 即表示“可不可 以”。例如:

A、我出要一下, 得行不得行嘛? （我可不可以出去 玩一下? ）

$B$ 、我现在得不得睡觉嘛？(我现在可不可以睡觉？) 


\section{5. 特殊句式}

\subsection{1. “陈述句+得嘛”}

通川方言中, “得嘛”置于陈述句句尾, 用来肯定已发 生的事情, 以此表达自己与对方之间的不同意见或看法。 陈述句前后偶尔带有与陈述的事实相悖的疑问句, 即使疑 问句不出现, 带“得嘛”的陈述句中也隐含着以陈述或肯定 事实所替代的反问语气。

其一，“得嘛”用在谓词之后。例如:

这个小品好看得嘛, 莫换台。（这个小品很好看, 不 要换台。）

我在写作业得啉, 等一哈就来。（我正在写作业, 等 一下就来。)

在以上的例句中, “得嘛”作为语气词, 放在谓词后面, 强调的是动作或状态。

其二, “ $\mathrm{V}$ 得”的否定句式是“ $\mathrm{V}$ 不得”, 其句尾也可以 用“得啉”。例如:

A、菜没熟, 还吃不得得嘛, 再等哈。（菜没熟, 还 不能吃, 再等一下。)

$\mathrm{B}$ 、我中午睡不得得嘛, 睡老半天睡不着。（我 中午不太好睡觉, 睡了好久也没睡着。)

其三, “得嘛”还可以用在“ $V+$ 得 $+C$ ”句式后面, 这里 的“ $V$ ”包含着能进入“ $\mathrm{V}+$ 得 $+\mathrm{C}$ ”句式的所有动词, 不论补语 是形容词还是动词, “得嘛” 都是句末语气词。例如:

A、你搬得动得嘛。(你能搬动嘛。)

$\mathrm{B} 、$ 这个菜看起来巴适得嘛。(这个菜看起来很好吃。)

其四, “得嘛”还能用在“ $V+$ 得 $+O$ ”句式之后。例如:

吃得苦得啉。( 能吃苦。)

其五, “ $\mathrm{V}+$ 得 $+\mathrm{O}$ ”否定式“ $\mathrm{V}+$ 不得 $+\mathrm{O}$ ”之后，也可以使 用“得嘛”。例如:

A、喝不得酒得嘛。(不能喝酒。)

B、吃不得辣椒得嘛。(不能吃辣椒。)

\subsection{2. “懒得 $+V$ ”}

“懒得”同“难得”，但与共同语的“难得”含义不同。共 同语的“难得”表示不容易办到或得到; 而通川方言中的 “懒得”或“难得”意义是消极的, 表示因有难度而不愿去做 或根本不会去做, 其后接动词。例如:

我才懒得管, 那个是他自己的事情。（我才不想管, 那是他自己的事情。)

这件事太麻烦, 我才懒得搞哦。（这件事太麻烦, 我 才不会去做。）

\subsection{3. “人称代词 + 不得了”}

“人称代词 + 不得了”中的“不得了”相当于“很”，常作 程度补语。例如:

针针鸡好切得不得了。（针针鸡太好吃了。）

\subsection{4. “喜幸得+陈述句”}

“幸喜得”表示“幸好、幸亏”之意，构成“喜幸得+陈述 句”句式。例如:

A、幸喜得我带了雨衣，没有淋到雨。

B、幸喜得他告诉我了, 不然我还在鼓里。（幸
好他告诉我了, 不然我还蒙在鼓里。)

\section{4. 结论}

“得”作为达州通川区的一种代表性方言现象, 在日常 交流中使用频率很高。通过分析，我们作一总结：

第一, 当“得 $\left[\mathrm{tie}^{214}\right]$ ”作助动词时, 表示有条件或有能 力做某事, 还表示某事有发生的可能性等意义; 但当它用 作结构助词时, 则无实在意义。

第二, 当“得 $\left[\mathrm{tie}^{35}\right]$ ”作动词时, 既有“获取”、“实现” 等义, 也含“有”之义; 而当它用作语气助词时, 其所表达 的语气丰富多样, 或表达陈述语气, 或表达强调语气, 等 等。

第三, “得”字的句式选择及句法特征, 诸如“V/adj+ 得”、“ $\mathrm{V}+$ 得 $+\mathrm{O}$ ”、“ $\mathrm{V} / \mathrm{adj}+$ 得 $+\mathrm{C}$ ”以及“喜幸得 + 陈述句”、“懒 得 $+\mathrm{V}+\mathrm{O}$ ”、“陈述句+得/得嘛”之类，可谓形式不同，作用 各异, 这也反映了当地方言的多姿多彩。

当然，还有如“得”字句的语用特征、其与周边（如成 都、自贡、广元等) 方言的异同等问题，均须作进一步的 分析研究。

\section{参考文献}

[1] 达州市志编纂委员会. 达州市志 [M]. 北京: 方志出版社, 2009:10。

[2] Qie Zhiyu. Sichuan Dialect and Culture[M]. Beijing: China international broadcasting press,2015:4.

[3] 张大旗.长沙话“得”字研究 [J].方言, 1985（1）。

[4] 徐阳春.南昌话“得”字研究[J].南昌大学学报,1998（4）。

[5] 陆镜光. 粤语“得”字的用法[J].方言,1999（3）。

[6] 袁莉容, 邓英树.四川方言中的情态助词“得”及其否定形式 [J].语言研究集刊(第十一辑), 2013 (2)。

[7] 邹欣芝.四川中江方言的“得”字研究[D].南昌大学硕士学位 论文, 2015:3,6。

[8] 杜军, 张潇月.四川方言“得”的语法化极其进程[J]. 重庆社会 科学, 2014 (3)。

[9] 李玲, 王玲娟.重庆话“没得”当议[J]. 南方文物, 2012（2）。

[10] Yin Runlin \&Xu Mei. Study of the Vocal Form "de" in Zigong Dialect[J]. Journal of Chengdu Electromechanical College, 2012(1)

[11] Ye Feisheng \&Xu Tongqiang. Linguistic Compendium[M]. Beijing: Peking University Press. 2010:151-154.

[12] 吕叔湘, 朱德熙.语法修辞讲话[M]. 北京: 中国青年出版社, 1952:19。

[13] 周家筠.成都话的“得”[ ]].四川大学学报( 哲社科版), 1981 (1)。 